\title{
Human migrating myoelectric complex in relation to gastrointestinal transit of a meal
}

\author{
J L Madsen, K Dahl
}

\begin{abstract}
Feeding interrupts the migrating myoelectric complex in most mammals. This study aimed to assess whether resumption of the migrating myoelectric complex in the human duodenum after eating was related to the gastrointestinal transit of the meal. Five healthy subjects participated in the study. After eating a radiolabelled test meal consisting of mixed liquid and solids, duodenal myoelectric activity and gastrointestinal transit of the meal were determined simultaneously. In spite of considerable variation in entire gastric emptying time between subjects (range $2 \cdot 5-5 \cdot 0$ hours), significant correlation was found between the completion of gastric emptying and the resumption of duodenal phase III activity within subjects $(p<0.01)$. A new technique for recording the duodenal myoelectric activity was used.
\end{abstract}

The migrating myoelectric complex is a specific pattern of electrical response activity that occurs cyclically in the small intestine of most mammals in the fasted state..$^{1-3}$ Migrating myoelectric complexes generally involve the duodenum and propagate distally. ${ }^{+}$After eating, the cycle is interrupted for a considerable period, depending on the composition of the meal. ${ }^{5 \cdot 7}$ We examined the possible association between gastric emptying and resumption of the duodenal myoelectric complex cycle activity, which has not been formally studied before.

\section{Materials and methods}

\section{RADIOLABELLED TEST MEAL}

The test meal consisted of $400 \mathrm{~g}$ of mixed solids and liquid $(80 \mathrm{~g}$ bread, $30 \mathrm{~g}$ cheese, $10 \mathrm{~g}$ butter, $50 \mathrm{~g}$ yoghurt, $230 \mathrm{~g}$ water) that had an energy value of $1600 \mathrm{~kJ}$. Two hundred $\mathrm{mg}$ of cellulose fibre labelled with $40 \mathrm{MBq}$ of technetium-99m was added to the yoghurt. The use of this radiolabelled marker in solid foods has been validated in a recent study. ${ }^{8}$

Department of Clinical Physiology and Nuclear Medicine and

Department of Clinical

Neurophysiology,

Rigshospitalet,

Copenhagen, Denmark

J L Madsen

K Dahl

Correspondence to:

Dr J L Madsen, Departmen of Clinical Physiology and Nuclear Medicine, Glostrup Hospital, DK-2600 Glostrup Denmark.

Accepted for publication 30 October 1989
DUODENAL MYOELECTRIC RECORDINGS

A new electrode technique was developed to record the duodenal myoelectric activity. The myoelectric signal was recorded with a single bipolar surface electrode (length $21 \mathrm{~mm}$, diameter $3 \mathrm{~mm}$, weight $0.6 \mathrm{~g}$ ) consisting of two stainless steel leading-off rings (width $7 \mathrm{~mm}$ ) which were mounted on the ends of a fitted teflon cylinder and connected to two recording wires (polyfile stainless steel, teflon coated, highly flexible, diameter $0.3 \mathrm{~mm}$ ) (Fig 1).

On the evening before the start of the investi- gations the electrode was introduced transnasally and allowed to advance until $90 \mathrm{~cm}$ of the recording wires were ingested. Next morning, as shown by fluoroscopy, the electrode was located in the distal half of duodenum.

The duodenal myoelectric signal was amplified (Dantec Counterpoint, upper frequency limit $1 \mathrm{kHz}$, lower frequency limit $20 \mathrm{~Hz}$, gain 50 $\mu \mathrm{V} /$ division) and recorded with an inkjet writer (Elema 34, upper frequency limit $1 \mathrm{kHz}$, 1 division $=1 \mathrm{~cm}$, overall gain $50 \mu \mathrm{V} / \mathrm{cm}$, paper speed $25 \mathrm{~mm} / \mathrm{second}$ ).

Five healthy subjects (four women, one man) with a mean age of 24 years (range 21-26) participated in the study after giving informed consent. The protocol for the studies had been previously approved by the local ethical committee. The studies began at $9.00 \mathrm{am}$ after an overnight fast. After having eaten the radiolabelled test meal within 10 minutes, the duodenal myoelectric activity was recorded continuously until the simultaneous gastrointestinal transit measurements had finished. Then the recording electrode was retrieved.

To reduce interference from striated muscle signals, subjects sat or stood quietly during the study.

Phase III activity was recognised by visual analysis as periods of uninterrupted regular electric response activity occurring at the rate of 10-12 per minute and lasting for longer than 2 minutes, followed by relative quiescence..$^{910} \mathrm{An}$ example of a phase III recording is shown in Figure 2. Any phase III activity recorded during the first 15 minutes after eating was omitted from the assessments as interruption of migrating myoelectric complex cycle might not have occurred during that period. ${ }^{10}$ To avoid subjective bias these analyses were performed before the gastrointestinal transit calculations.

\section{GASTROINTESTINAL TRANSIT MEASUREMENTS}

Gastrointestinal transit measurements were carried out as described previously. ${ }^{8}$ Immediately after eating the radiolabelled test meal, imaging was performed with the subject in the upright position. A large field of view gamma camera (General Electric MaxiCamera 535, medium energy, parallel hole collimator) was used. Anterior and posterior images were

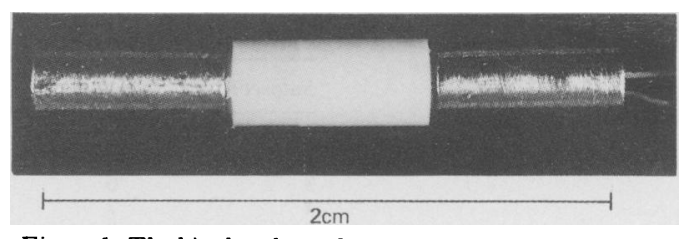

Figure 1: The bipolar electrode. 

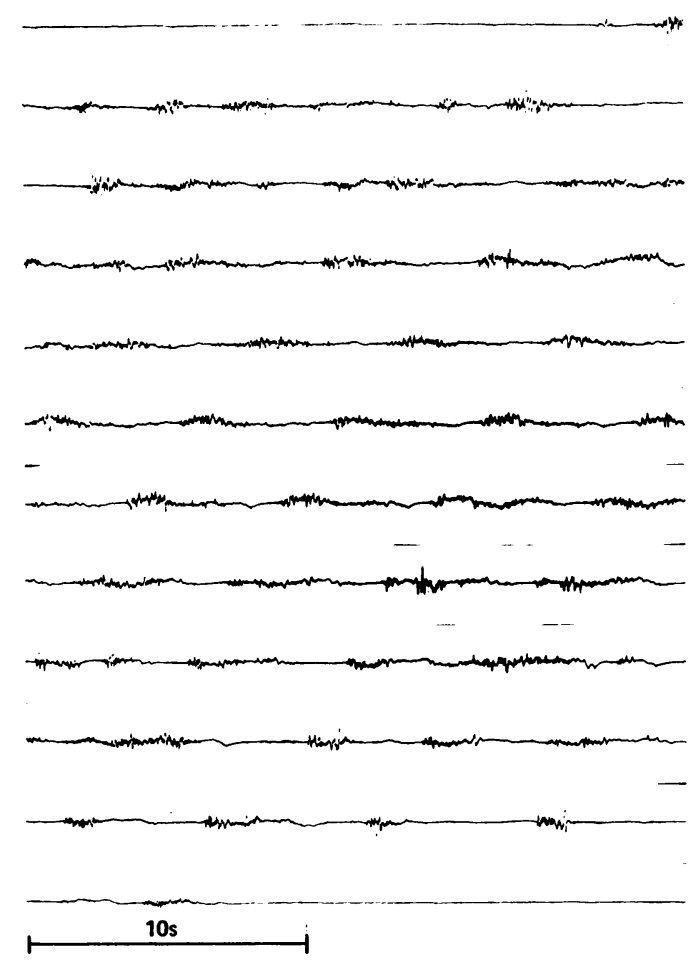

Figure 2: Representative tracing of duodenal phase III activity.

obtained using a $15 \%$ energy window over the $140-\mathrm{keV}^{99 \mathrm{~m}} \mathrm{Tc}$ photopeak. Each imaging period lasted two minutes. Imaging was repeated at $\mathbf{3 0}$ minute intervals until no activity could be detected in the small intestine. Subjects were sitting between imaging. Data were stored on a computer for later analysis.

\section{CALCULATIONS}

Regions of interest for integration of activity were delineated manually around the stomach, the small intestine, and colon on each image. The obtained count rates were corrected for physical decay. The geometric means of corresponding anterior and posterior count rates were used as attenuation corrections." The corrected count rates were then converted into fractions of the maximum count rate. For each gastrointestinal segment, these fractions were plotted against time. The moment that the first phase III migrating myoelectric complex occurred was marked out on these curves. The corresponding amount of activity in each segment was then read by interpolation from the curves. In addition, the entire gastric emptying time and the corresponding phase III interruption time were compared by linear regression. The obtained correlation coefficient was tested against 0 .

The percentage distribution of radiolabelled cellulose fibre in the various gastrointestinal segments of five subjects at resumption of duodenal phase III activity after a meal. (For comparison with Fig 3, subjects are ranked according to phase III interruption time. Rank I means shortest interruption time)

\begin{tabular}{llcc}
\hline Subject & Stomach & Small intestine & Colon \\
\hline 1 & 2 & 88 & 10 \\
2 & 0 & 100 & 0 \\
3 & 0 & 41 & 59 \\
4 & 7 & 79 & 14 \\
5 & 0 & 45 & 55 \\
\hline
\end{tabular}

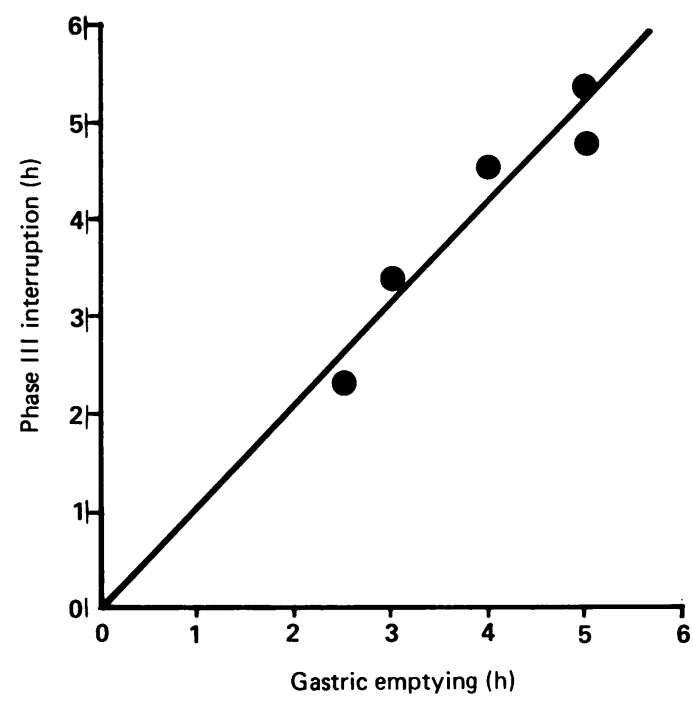

Figure 3: The entire gastric emptying time of five subjects plotted against the corresponding phase III interruption time after a meal. The regression line is drawn.

\section{Results}

Results of the gastrointestinal transit measurements and the myoelectric recordings are shown in the Table and in Figure 3.

Linear regression showed a significant correlation between the entire gastric emptying time and the corresponding phase III interruption time (correlation coefficient $0.96, p<0.01$, slope of regression line $1 \cdot 04$, intercept on $y$-axis $0 \cdot 04$ ).

Obviously, a correlation did not exist between resumption of phase III activity and the corresponding content of radiolabelled marker in the small intestine or colon.

\section{Discussion}

The migrating myoelectric complex cycle or its motor correlate in the human small intestine is interrupted by a meal. The duration of this interruption is proportional to the calorific value of the meal and the composition of the food ingested. Lipids interrupt the migrating myoelectric complex cycle for longer than carbohydrates and proteins of equal calorific value..$^{12}$ As gastric emptying of larger meals is delayed compared with that of smaller meals and lipids are emptied more slowly than non-lipids, ${ }^{1+15}$ the interruption of the migrating myoelectric complex cycle may be related to the gastrointestinal transit of the meals. Such a relation has not yet, however, been described precisely.

This study showed a very close temporal association between the end gastric emptying and the resumption of the duodenal migrating myoelectric complex cycle. The cause of this association is not clear as the control mechanisms involved in the migrating myoelectric complex cycle have not been fully explained. ${ }^{36}$ Our results, however, can probably be explained by the hypothesis that when duodenal chemoreceptors are no longer stimulated, cycle activity controlled by the enteric nervous system is unblocked.

The considerable variation in the time that the first phase III activity occurs after a meal reported earlier ${ }^{+9}$ may, as in this report, reflect the variation in gastric emptying kinetics. 
The physiological role of the migrating myoelectric complex and the corresponding motor activity is still unknown. Our results possibly support the thesis that the cycle is important in propelling residual food along the small intestine since this segment contained considerable amounts of the food marker when the migrating myoelectric complex cycle recurred after feeding.

This work was supported by the Danish Hospital Foundation fo Medical Research; Region of Copenhagen, The Faroe Islands and Greenland.

1 Szurszewski JH. A migrating electric complex of the canine small intestine. Am F Physiol 1969; 217: 1757-63.

2 Fleckenstein P. Migrating electrical spike activity in the fasting human small intestine. Am f Dig Dis 1978; 23: 26975.

3 Wingate DL. Backwards and forwards with the migrating complex. Dig Dis Sci 1981; 26: 641-66.

4 Kellow JE, Borody TJ, Phillips SF, Tucker RL, Haddad AC Human interdigestive motility: variations in patterns from esophagus to colon. Gastroenterology 1986; 91: 386-95.

5 Code CF, Marlett JA. The interdigestive myoelectric complex of the stomach and small bowel of dogs. $\mathcal{F}$ Physiol 1975; 246 : 289-309.
6 Sarna SK. Cyclic motor activity; migrating motor complex: 1985. Gastroenterology 1985; 89: 894-913.

7 Eeckhout C, De Wever I, Vantrappen G, Janssens J. Local disorganization of interdigestive migrating complex by perfusion of a Thiry-Vella loop. Am $f$ Physiol 1980, 238:

8 Madsen JL, Jensen M. Gastrointestinal transit of technetium $99 \mathrm{~m}$-labeled cellulose fiber and indium-111-labeled plastic particles. F Nucl Med 1989; 30: 402-6.

9 Thom WJ, Hardy RJ. Normal patterns of human upper smal bowel motor activity recorded by prolonged radiotelemetry. Gut 1980; 21 : $500-6$.

10 Houghton LA, Read NW, Heddle F, et al. Motor activity of the gastric antrum, pylorus, and duodenum under fasted conditions and after a liquid meal. Gastroenterology 1988; 94: 1276-84.

11 Hardy JG, Perkins AC. Validity of the geometric mean correction in the quantification of whole bowel transit. Nucl Med Commun 1985; 6: 217-24.

12 De Wever I, Eeckhout C, Vantrappen G, Hellemans J. Disruptive effect of test meals on interdigestive motor

13 Rees WDW, Malagelada J-R, Miller JL, Go VLW. Human interdigestive and postprandial gastrointestinal motor and gastrointestinal hormone patterns. Dig Dis Sci 1982; 27 321-9.

14 Read NW, Cammack J, Edwards C, Holgate AM, Cann PA Brown C. Is the transit time of a meal through the small intestine related to the rate at which it leaves the stomach? Gut 1982; 23: 824-8.

15 Jian R, Vigneron N, Najean Y, Bernier JJ. Gastric emptying and intragastric distribution of lipids in man. Dig Dis $S c i$ 1982; 27: 705-11. 